\title{
Optimization of a Low Magnesium, Cholesterol-Containing Diet for the Development of Atherosclerosis in Rabbits
}

\author{
Brendon W. Smith ${ }^{1,2}$, Jennifer L. King ${ }^{1}$, Rita J. Miller ${ }^{1}$, James P. Blue Jr. ${ }^{1}$, Sandhya Sarwate ${ }^{1,3}$, William D. \\ O’Brien Jr. ${ }^{1,2}$ \& John W. Erdman Jr. ${ }^{2,4}$ \\ ${ }^{1}$ Bioacoustics Research Laboratory, Department of Electrical and Computer Engineering, University of Illinois, \\ Urbana-Champaign, IL, USA \\ ${ }^{2}$ Division of Nutritional Sciences, University of Illinois, Urbana-Champaign, IL, USA \\ ${ }^{3}$ Department of Pathology, University of Illinois, Urbana-Champaign, IL, USA \\ ${ }^{4}$ Department of Food Science and Human Nutrition, University of Illinois, Urbana-Champaign, IL, USA \\ Correspondence: John W. Erdman Jr., Department of Food Science and Human Nutrition, University of Illinois, \\ Urbana-Champaign, 455 Bevier Hall, 905 South Goodwin Avenue, Urbana, IL 61801, USA. Tel: \\ 1-217-333-2527. E-mail: jwerdman@illinois.edu
}

Received: November 12, 2012 Accepted: December 5, 2012 Online Published: January 28, 2013

doi:10.5539/jfr.v2n1p168 URL: http://dx.doi.org/10.5539/jfr.v2n1p168

\begin{abstract}
The cholesterol-fed rabbit is useful for atherosclerosis research. We describe development of a low-magnesium $(\mathrm{Mg})$ cholesterol-containing diet to accelerate atherosclerosis in this model. Male New Zealand White rabbits were fed either chow or one of four atherogenic diets: $1 \%$ cholesterol $10 \%$ fat $0.11 \% \mathrm{Mg}, 1 \%$ cholesterol $10 \%$ fat $0.40 \% \mathrm{Mg}, 2 \%$ cholesterol $20 \%$ fat $0.11 \% \mathrm{Mg}$, or $2 \%$ cholesterol $20 \%$ fat $0.40 \% \mathrm{Mg}$. While feed intake decreased in cholesterol-fed rabbits, they were able to maintain their body weights. Rabbits consuming cholesterol experienced profound hypercholesterolemia and tissue lipid accumulation, with plasma cholesterol levels above $1500 \mathrm{mg} / \mathrm{dl}$ for all groups at the completion of the study. Liver and spleen lipid content and liver cholesterol content also increased. Aortic arch atheroma thickness was greatest in 1\% cholesterol $10 \%$ fat $0.11 \%$ $\mathrm{Mg}$ animals. Tissue Mg levels decreased in cholesterol-fed animals compared to chow-fed controls, despite equal or greater serum $\mathrm{Mg}$ levels. Our results indicate that the $1 \%$ cholesterol $10 \%$ fat $0.11 \% \mathrm{Mg}$ diet was optimal at promoting hypercholesterolemia and atherosclerosis while minimizing health complications for the animals. The low $\mathrm{Mg}$ cholesterol diet will be useful to other biomedical researchers interested in utilizing the rabbit for cardiovascular disease research.
\end{abstract}

Keywords: atherosclerosis, cardiovascular diseases, cholesterol, magnesium, rabbits

Abbreviations: ANOCOVA, Analysis of Covariance; ANOVA, Analysis of Variance; CVD, cardiovascular disease; LDL, low-density lipoprotein; VLDL, very low-density lipoprotein; Mg, Magnesium; RBC, red blood cell; vWF, von Willebrand Factor

\section{Introduction}

Cardiovascular Disease (CVD) remains a major public health burden and a leading cause of death worldwide (Roger et al., 2011). A gradual, silent pathological process known as atherosclerosis underpins the progression of CVD. Our current understanding suggests that an immune response to ectopic low-density lipoprotein (LDL) deposits in the blood vessel wall drives the formation and development of atherosclerotic plaques (Hansson \& Hermansson, 2011). Rupture of these plaques is manifested in cardiovascular events including myocardial infarction and stroke.

Animal models have proven critical in advancing our understanding of atherosclerosis. Numerous animal models have been used, including monkeys, pigs, rabbits, hamsters, and genetically modified mice. Among these, rabbits are the most well established and have been in use since the early 1900s (Finking \& Hanke, 1997). Rabbits are highly sensitive to dietary cholesterol and develop severe hypercholesterolemia and atherosclerosis when it is introduced into their diets. While cholesterol feeding is sufficient for development of atherosclerotic lesions in rabbits, it requires studies of prolonged duration. Animal housing, feeding, and other per diem costs are 
expensive, and it is highly advantageous for funding agencies and biomedical researchers alike to generate results in the least possible time.

Adjustment of the composition of the rabbit diet could yield an acceleration of atherosclerosis. In addition to cholesterol, magnesium $(\mathrm{Mg})$ is another dietary component that can be adjusted to more rapidly effect atherosclerosis. Mg intake is inversely correlated with risk of CVD and CVD-related mortality in humans (Song \& Liu, 2012), and atherosclerosis is reduced in cholesterol-fed rabbits supplemented with Mg (Ouchi et al., 1990). We hypothesized that a reduction in dietary Mg would lead to rapid development of hypercholesterolemia and atherosclerosis in rabbits. We previously published a preliminary report of our animal model development (King et al., 2009). Here we present an expanded description of development of the low Mg atherosclerotic diet for rabbits.

\section{Materials and Methods}

\subsection{Animals and Diets}

Four-month-old male New Zealand White rabbits (Myrtle's Rabbitry, Thompson's Station, TN) with initial body weights between 3.05-3.75 kg were housed individually in standard caging with stainless steel mesh bottom at normal temperature and light cycles. All procedures were approved by the Institutional Animal Care and Use Committee at the University of Illinois at Urbana-Champaign.

After an acclimation period of one week on standard chow diet (2031 Global High Fiber Rabbit Diet, Harlan ${ }^{\circledR}$ Teklad, Indianapolis, IN), rabbits ( $\mathrm{n}=2-16$ per group per timepoint) were fed either chow or one of four semi-purified pelleted atherogenic diets (TestDiet ${ }^{\mathbb{\mathbb { R }}}$, Richmond, IN): $1 \%$ cholesterol $10 \%$ fat $0.11 \% \mathrm{Mg}$ (5TZB), $1 \%$ cholesterol $10 \%$ fat $0.40 \% \mathrm{Mg}$ (5TZD), $2 \%$ cholesterol $20 \%$ fat $0.11 \% \mathrm{Mg}$ (5TSV), or $2 \%$ cholesterol, $20 \%$ fat, $0.40 \% \mathrm{Mg}(5 \mathrm{TSW})$. The complete nutritional composition of the diets is provided in Table 1. A small amount of soybean oil was included in the diets to prevent essential fatty acid deficiency. Rabbits were gradually introduced to the atherogenic diets over ten days by replacing $10 \%$ of the chow with atherogenic diet each day until the rabbits were consuming only the atherogenic diet on the tenth day. Rabbits were randomly assigned to diets, offered between 140-300 g of feed daily depending on feed intake and provided with tap or deionized water ad libitum. Apple slices or sugar water was provided as necessary to encourage feed intake. Animals that did not consume feed for 72 hours or more were excluded from data analysis. Feed intake was measured daily, fresh feed was also provided daily and body weights were measured weekly. Samples of each diet were analyzed by an independent laboratory (Waters Agricultural Laboratories, Camilla, GA) to verify protein, fat, fiber and Mg content.

Blood samples were obtained at regular timepoints throughout the study via the saphenous or jugular veins while under restraint. Sodium heparin was used to prevent clotting for blood plasma isolation. Blood was then centrifuged at $1380 \mathrm{xg}$ and room temperature for 10 minutes and plasma was aliquotted and frozen at $-70^{\circ} \mathrm{C}$. The pelleted cellular fraction left after removal of plasma was washed with $0.9 \%$ saline and centrifuged at $150 \mathrm{xg}$ for 10 minutes. The supernatant was discarded and this procedure was repeated twice until the supernatant was colorless. The cellular fraction, containing red blood cells (RBCs), was then frozen at $-70^{\circ} \mathrm{C}$.

After consuming their respective diets for eight weeks, animals were euthanized by $\mathrm{CO}_{2}$ asphyxiation under anesthesia. Aortas, livers, spleens, hearts, and skeletal muscle tissue (abdominal or leg) were removed. Aortas were fixed in formalin, and livers, spleens, hearts, and skeletal muscle tissue were frozen at -20 or $-70^{\circ} \mathrm{C}$ until analysis. Liver lipid extraction was performed using a modified Folch method as previously described (Smith et al., 2012). 
Table 1. Composition of cholesterol-containing diets

\begin{tabular}{|c|c|c|c|c|c|}
\hline \multirow{3}{*}{ Dietary Component } & \multirow{3}{*}{ Chow } & \multirow{3}{*}{$\begin{array}{l}1 \% \text { cholesterol } \\
10 \% \text { fat } \\
0.11 \% \mathrm{Mg}\end{array}$} & \multirow{3}{*}{$\begin{array}{l}1 \% \text { cholesterol } \\
10 \% \text { fat } \\
0.40 \% \mathrm{Mg}\end{array}$} & \multirow{3}{*}{$\begin{array}{l}2 \% \text { cholesterol } \\
20 \% \text { fat } \\
0.11 \% \mathrm{Mg}\end{array}$} & \multirow{3}{*}{$\begin{array}{l}2 \% \text { cholesterol } \\
20 \% \text { fat } \\
0.40 \% \mathrm{Mg}\end{array}$} \\
\hline & & & & & \\
\hline & & & & & \\
\hline Energy, kcal/g & 1.7 & 3.01 & 3.01 & 3.65 & 3.79 \\
\hline Protein, \% & 14 & 14 & 14 & 15 & 15 \\
\hline Total Fat, \% & 2.7 & 10 & 10 & 20 & 20 \\
\hline Total SFA, \% & 0.71 & 2.0 & 2.0 & 4.5 & 4.5 \\
\hline Total MUFA, $\%$ & 0.67 & 2.7 & 2.7 & 5.9 & 6.0 \\
\hline Total PUFA, \% & 1.2 & 3.2 & 3.1 & 5.5 & 5.6 \\
\hline Carbohydrate, $\%$ & 43 & 53 & 53 & 35 & 35 \\
\hline Fiber, $\%$ & 21 & 21 & 21 & 17 & 17 \\
\hline Cholesterol, \% & 0 & 1 & 1 & 2 & 2 \\
\hline $\mathrm{Mg}, \%$ & 0.19 & 0.11 & 0.40 & 0.11 & 0.40 \\
\hline $\mathrm{Ca}, \%$ & 1.1 & 0.90 & 0.89 & 0.90 & 0.89 \\
\hline $\mathrm{P}, \%$ & 0.58 & 0.70 & 0.70 & 0.70 & 0.70 \\
\hline $\mathrm{K}, \%$ & 1.1 & 1.2 & 1.2 & 1.2 & 1.2 \\
\hline $\mathrm{Na}, \%$ & 0.31 & 0.31 & 0.30 & 0.32 & 0.30 \\
\hline $\mathrm{Cl}, \%$ & 0.70 & 0.64 & 0.64 & 0.64 & 0.64 \\
\hline
\end{tabular}

Ingredients are listed as percent by weight. Cholesterol diets contained alfalfa meal, cellulose, cornstarch, corn, dehulled soybean meal, Crisco, wheat gluten, casein, corn flour, potassium phosphate, soybean oil, cane molasses, oats, calcium carbonate, cholesterol, salt, vitamin/mineral premix, artificial flavors, choline chloride, DL-methionine, L-arginine, L-threonine, and L-tryptophan. Magnesium oxide was added to the $0.40 \% \mathrm{Mg}$ diet. All diets were pelleted, and fat and cholesterol were added by TestDiet ${ }^{\circledR}$ during the pelleting process. The nutritional profiles for the most recent lots used are given. SFA, Saturated Fatty Acids; MUFA, Monounsaturated Fatty Acids; PUFA, Polyunsaturated Fatty Acids.

\subsection{Cholesterol and $v W F$}

Plasma and liver total cholesterol was measured using an enzymatic colorimetric kit (Wako, Richmond, VA) according to the manufacturer's instructions, with samples plated in triplicate and human control sera (Wako) used as internal controls in each assay. The Coefficient of Variation (CV) was calculated using the equation

$$
C V=(\text { Standard Deviation/Mean }) \times 100
$$

Intra-assay CVs were $8.3 \%$ for rabbit samples and $9.9 \%$ for control sera, and the inter-assay CV for human control sera was $10 \%$, indicating that the assay was consistently precise. vWF was measured by sandwich ELISA in triplicate as previously described (Smith et al., 2012). The average intra-assay CV was $5.1 \%$.

\subsection{Histology}

Formalin-fixed $3 \mu \mathrm{m}$ transverse sections of the aortic arch were stained with $\mathrm{H} \& \mathrm{E}$ and evaluated by a board-certified pathologist blinded to the experimental conditions as previously described (Smith et al., 2012). Atheroma thickness was measured at the thickest point in the aortic arch using an ocular micrometer (Olympus America Inc., Center Valley, PA). An atherosclerosis score was defined between 0 and 5 using the American Heart Association classification scheme for human atherosclerotic lesions (Kumar et al., 2004). Score $0=$ Absence of atherosclerosis; Score 1 = Presence of isolated foam cells; Score 2 = Lipid accumulation mainly within the foam cells; Score 3 = Lipid accumulation within the foam cells and small pools of extracellular lipid; Score 4 = Intracellular lipid, lipid pools and core of extracellular lipid; and Score 5 = Lipid core and fibrotic layer, or multiple lipid cores and fibrotic layer, or mainly calcified or fibrotic plaque.

\section{$2.4 \mathrm{Mg}$ Analysis}

Serum Mg levels were determined on a clinical diagnostic machine (Veterinary Diagnostic Laboratory, College 
of Veterinary Medicine, University of Illinois at Urbana-Champaign). Mg levels in RBCs, cardiac and skeletal muscle were determined by inductively coupled plasma-optical emission spectroscopy (University of Missouri Agricultural Experiment Station Chemical Laboratories, Columbia, MO).

\subsection{Statistical Analysis}

Statistical analysis was conducted in SAS 9.3 for Windows, and graphs were created in SigmaPlot 12.0 for Windows. Comparisons between two groups were conducted with an independent samples T-test. Normality was assessed using a Shapiro-Wilk test in the univariate procedure of SAS, and homogeneity of variances was confirmed within the T-test procedure. Data that did not meet the assumptions of the T-test were analyzed using the Wilcoxon rank sum test. Comparisons among three or more groups were made using one-way analysis of variance (ANOVA) with a Tukey's test for multiple comparisons within the general linear models procedure. For ANOVA testing, normality was determined as described above, and homogeneity of variances was determined using a Brown-Forsythe test. Data that did not meet the normality assumption of the one-way ANOVA were analyzed using the non-parametric Kruskal-Wallis test followed by a Dunn's test for multiple comparisons. Analyses of serial measurements (feed intake, body weights, plasma cholesterol, plasma vWF, serum Mg and red blood cell $\mathrm{Mg}$ ) were performed using a repeated measures analysis of covariance (ANOCOVA) in the mixed models or general linear mixed models procedures of SAS. Timepoint and diet were used as the major fixed effects, and baseline measurements were used as a covariate. When significant differences among groups within timepoints were noted, multiple comparisons were then performed using estimate statements based on the least squares means, followed by Bonferroni adjustment for multiplicity. Normality was assessed as described above. The covariance structure of repeated measures data was assessed both graphically and quantitatively and an appropriate covariance model was fit. The threshold for declaring statistical significance was set at $\mathrm{p}<0.05$.

\section{Results}

\subsection{Feed Intake, Body Weights and Animal Health}

Feed intake significantly decreased after three weeks for all animals consuming cholesterol, and this decrease persisted until the end of the study ( $<0.05$ by mixed models repeated measures ANOCOVA, Figure 1A).While body weights differed among groups within the first three weeks of the study $(\mathrm{p}<0.05$ by mixed models repeated measures ANOCOVA, Figure 1B), this significance was not sustained in the subsequent five weeks, and animals were able to maintain their body weights despite reduced feed intake. An additional dataset was generated by calculating the change in body weight (final-initial) for each animal in the study. There were no significant differences in change in body weight after the eight weeks of the study among the five diet groups $(\mathrm{p}>0.05$ by one-way ANOVA with Tukey's test for multiple comparisons). Incidence of anorexia (defined as complete lack of feed intake for 72 hours or more) was $50 \%$ in animals fed $2 \%$ cholesterol $20 \%$ fat $0.11 \% \mathrm{Mg}, 25 \%$ in animals fed $1 \%$ cholesterol $10 \%$ fat $0.40 \% \mathrm{Mg}, 13 \%$ in animals fed $2 \%$ cholesterol $20 \%$ fat $0.40 \% \mathrm{Mg}$ and $12 \%$ in animals fed $1 \%$ cholesterol $10 \%$ fat $0.11 \% \mathrm{Mg}$. Anorexic animals were excluded from data analysis, which reduced the number of timepoints with available data for the $2 \%$ cholesterol $20 \%$ fat groups. Incidence of jaundice, as assessed by visual yellowing of ears, skin or eyes, was $30 \%$ in the $1 \%$ cholesterol $10 \%$ fat $0.40 \%$ Mg group, $25 \%$ in animals fed $2 \%$ cholesterol $20 \%$ fat $0.40 \% \mathrm{Mg}$ and $12 \%$ in animals fed $1 \%$ cholesterol $10 \%$ fat $0.11 \% \mathrm{Mg}$. No jaundice was observed in animals fed $2 \%$ cholesterol $20 \%$ fat $0.11 \% \mathrm{Mg}$. Premature death was noted in $3 \%$ of the total animals ( $2 / 58$ rabbits) in the $1 \%$ cholesterol $10 \%$ fat $0.11 \% \mathrm{Mg}$ group. 

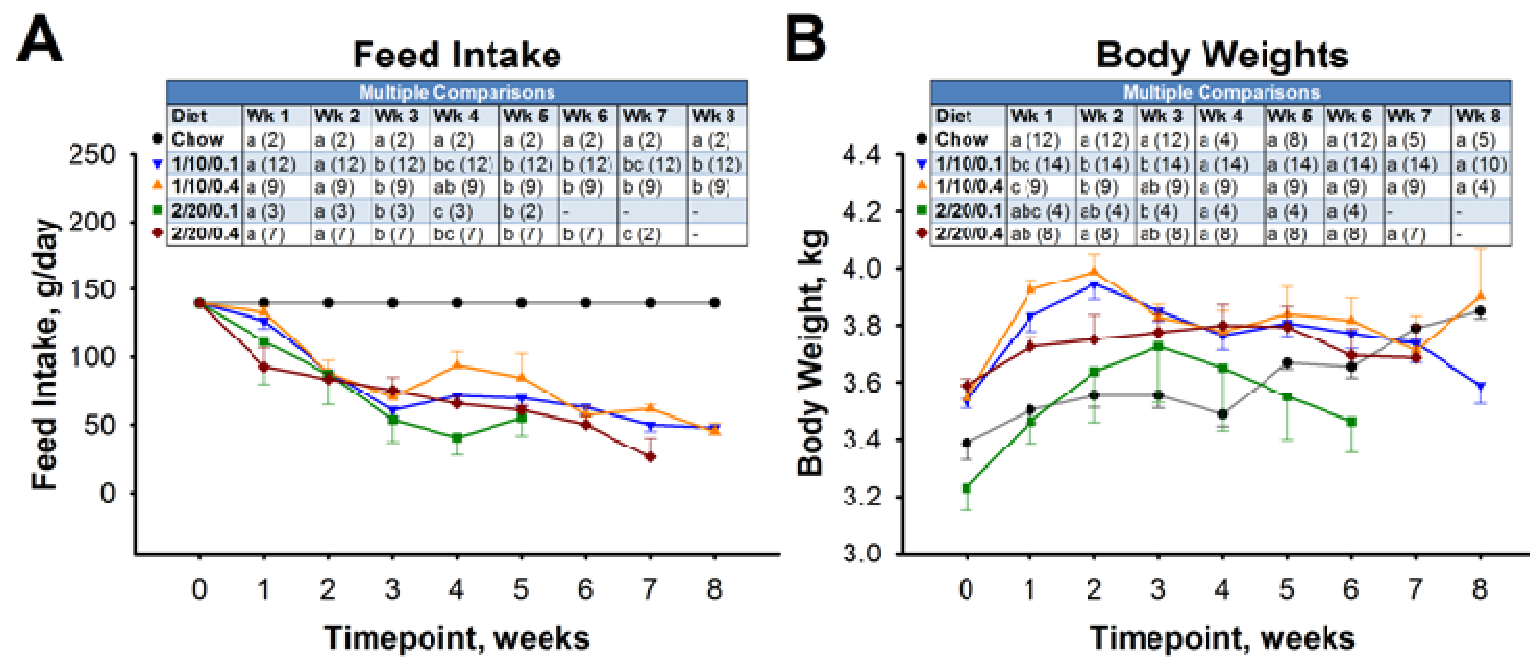

Figure 1. Feed intake (A) and body weights (B)

Four month old rabbits ( $\mathrm{n}=2-14$ per group per timepoint) were randomized to consume one of five diets: standard chow diet (Chow), $1 \%$ cholesterol $10 \%$ fat $0.11 \% \mathrm{Mg}(1 / 10 / 0.1), 1 \%$ cholesterol $10 \%$ fat $0.40 \% \mathrm{Mg}$ (1/10/0.4), $2 \%$ cholesterol $20 \%$ fat $0.11 \% \mathrm{Mg}(2 / 20 / 0.1)$, or $2 \%$ cholesterol, $20 \%$ fat, $0.40 \% \mathrm{Mg}(2 / 20 / 0.4)$. Timepoint 0 indicates a baseline measurement prior to initiation of the atherogenic diet. Rabbits were gradually introduced to the atherogenic diets over ten days by replacing $10 \%$ of the chow with atherogenic diet each day until the rabbits were consuming only the atherogenic diet on the tenth day. The week 1 timepoint begins on the tenth day and represents the first week rabbits were exclusively consuming the atherogenic diet. Feed intake was measured daily and body weights were measured weekly. Bars are SEM. Data were analyzed using a mixed models repeated measures ANOCOVA adjusted for baseline measurements, and multiple comparisons within timepoints among diet groups were performed using least squares means estimate statements with Bonferroni adjustment for multiplicity. Means not sharing a letter are significantly different at the $\mathrm{p}<0.05$ level, and sample sizes are provided in parentheses. $\mathrm{Mg}$, magnesium.

\subsection{Plasma Cholesterol and $v W F$}

Plasma cholesterol levels in chow-fed animals averaged $21 \mathrm{mg} / \mathrm{dl}$ during the study, but increased significantly in all animals consuming cholesterol (Figure 2A). Animals in the $2 \%$ cholesterol $20 \%$ fat $0.40 \% \mathrm{Mg}$ group experienced the most rapid elevation in plasma cholesterol levels, reaching an average of $1597 \mathrm{mg} / \mathrm{dl}$ by week 6 . By 8 weeks all animals in the $1 \%$ cholesterol $10 \%$ fat groups also had similar plasma cholesterol levels, averaging $1719 \mathrm{mg} / \mathrm{dl}$ in the $0.11 \% \mathrm{Mg}$ animals and $1690 \mathrm{mg} / \mathrm{dl}$ in the $0.40 \% \mathrm{Mg}$ animals.

vWF levels were not significantly different at baseline among the three groups, but increased in the two cholesterol-fed groups during the study ( $\mathrm{p}<0.05$ by mixed models repeated measures ANOCOVA, Figure 2B). After 8 weeks on their respective diets, vWF averaged $4200 \mathrm{ng} / \mathrm{ml}$ in chow-fed animals, $8400 \mathrm{ng} / \mathrm{ml}$ in animals fed $1 \%$ cholesterol $10 \%$ fat $0.11 \% \mathrm{Mg}$, and $13000 \mathrm{ng} / \mathrm{ml}$ in animals fed $1 \%$ cholesterol $10 \%$ fat $0.40 \% \mathrm{Mg}$. 

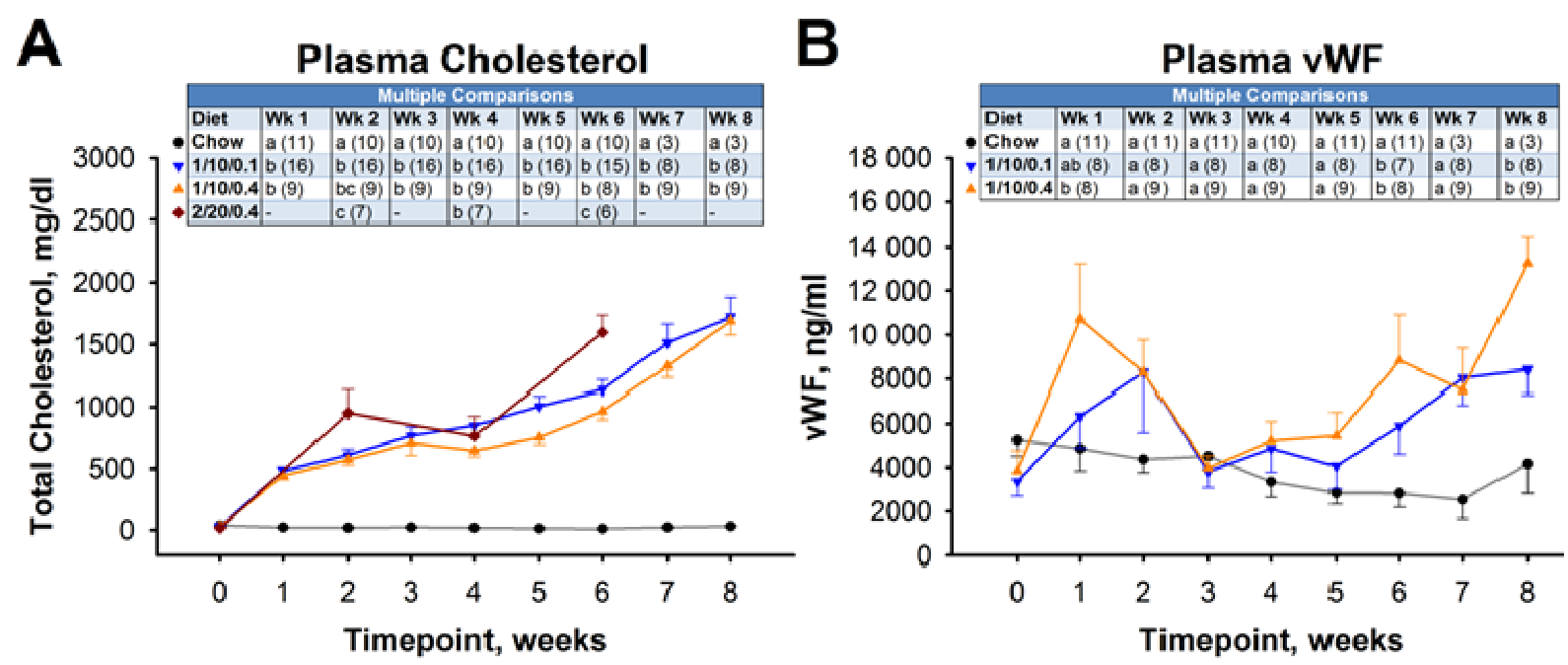

Figure 2. Plasma total cholesterol (A) and von Willebrand Factor (B)

Blood samples were collected from the lateral saphenous vein while under restraint at the timepoints indicated. See Figure 1 caption for diet abbreviations. Timepoint 0 indicates a baseline measurement prior to initiation of the atherogenic diet. For animals in the $2 / 20 / 0.4 \mathrm{Mg}$ group, blood was collected prior to initiation of the atherogenic diet (timepoint 0) and weeks 2,4 and 6. Blood was not collected from animals in the 2/20/0.1 group. Blood plasma was isolated, and total cholesterol levels were determined by enzymatic colorimetric kit $(\mathrm{n}=3-16$ per group per timepoint). von Willebrand Factor (vWF) levels were determined for the chow, 1/10/0.1 and 1/10/0.4 groups using a sandwich ELISA ( $n=3-11$ per group per timepoint). Bars are SEM. Data were analyzed using a mixed models repeated measures ANOCOVA on log transformed data and adjusted for baseline measurements, and multiple comparisons within timepoints among diet groups were performed using least squares means estimate statements with Bonferroni adjustment for multiplicity. Means not sharing a letter are significantly different at the $\mathrm{p}<0.05$ level, and sample sizes are provided in parentheses.

\subsection{Liver and Spleen Lipids and Liver Cholesterol}

At termination of the study, cholesterol accumulation in livers of both $0.11 \% \mathrm{Mg}$ and $0.40 \% \mathrm{Mg}$-fed rabbits was significantly greater than chow-fed controls ( $\mathrm{p}<0.01$ by one-way ANOVA with Tukey's test for multiple comparisons), averaging $2.6 \mathrm{mg} / \mathrm{g}$ in chow-fed animals, $5.1 \mathrm{mg} / \mathrm{g}$ in animals fed $1 \%$ cholesterol $10 \%$ fat $0.11 \%$ $\mathrm{Mg}$, and $4.6 \mathrm{mg} / \mathrm{g}$ in animals fed $1 \%$ cholesterol $10 \%$ fat $0.40 \% \mathrm{Mg}$ (Figure 3A). Lipid accumulation in livers of both $0.11 \% \mathrm{Mg}$ and $0.40 \% \mathrm{Mg}$-fed rabbits was also significantly greater than chow-fed controls $(\mathrm{p}<0.05$ by one-way ANOVA with Tukey's test), averaging $60 \mathrm{mg} / \mathrm{g}$ in chow-fed animals, $250 \mathrm{mg} / \mathrm{g}$ in animals fed $1 \%$ cholesterol $10 \%$ fat $0.11 \% \mathrm{Mg}$, and $240 \mathrm{mg} / \mathrm{g}$ in animals fed $1 \%$ cholesterol $10 \%$ fat $0.40 \% \mathrm{Mg}$ (Figure 3B). Lipid accumulation in spleens of both $0.11 \% \mathrm{Mg}$ and $0.40 \% \mathrm{Mg}$-fed rabbits was significantly greater than chow-fed controls ( $\mathrm{p}<0.05$ by one-way ANOVA with Tukey's test), averaging $32 \mathrm{mg} / \mathrm{g}$ in chow-fed animals, 130 $\mathrm{mg} / \mathrm{g}$ in animals fed $1 \%$ cholesterol $10 \%$ fat $0.11 \% \mathrm{Mg}$, and $120 \mathrm{mg} / \mathrm{g}$ in animals fed $1 \%$ cholesterol $10 \%$ fat $0.40 \% \mathrm{Mg}$ (Figure 3C). 

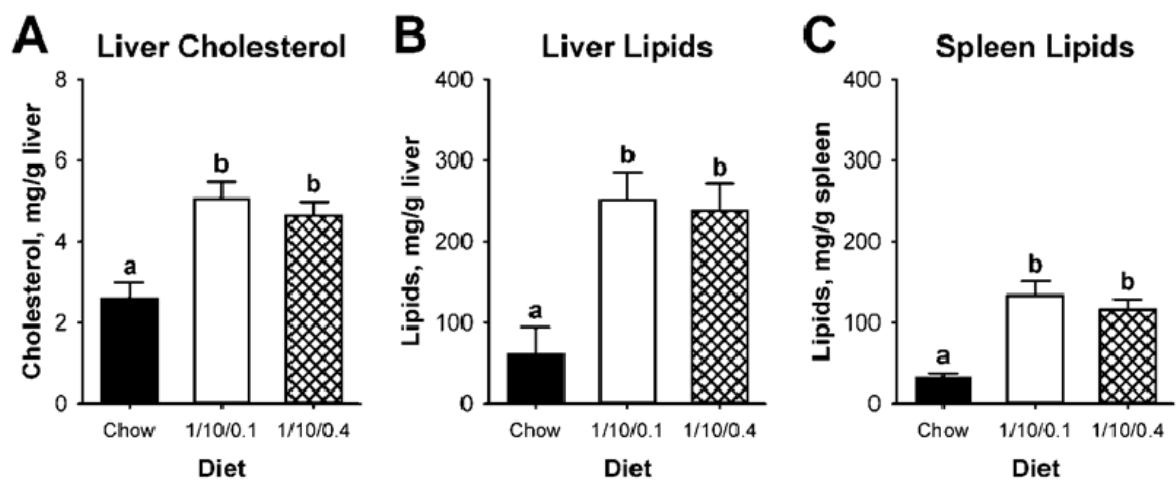

Figure 3. Tissue lipid and cholesterol accumulation in cholesterol-fed rabbits. Rabbits were euthanized after 8 weeks on their diets

Liver (A and B) and spleen lipids (C) were extracted using a modified Folch method (chown=3, 1/10/0.1 $\mathrm{n}=8$, and $1 / 10 / 0.4 n=9$ per group). Bars are SEM. See Figure 1 caption for diet abbreviations. Lipid and cholesterol content were not measured for animals in the 2/20/0.4 and 2/20/0.1 groups. Means not sharing a letter are significantly different $(\mathrm{p}<0.01$ for liver cholesterol and $\mathrm{p}<0.05$ for liver and spleen lipids by one-way ANOVA with Tukey's test).

\subsection{Histology}

$\mathrm{H} \& \mathrm{E}$ stained sections from rabbits after 8 weeks on the atherogenic diets showed thick atheromas containing abundant foam cells engorged with lipid (Figure 4A and 4B). Aortic arch atheroma thickness averaged $360 \mu \mathrm{m}$ in animals fed $1 \%$ cholesterol $10 \%$ fat $0.11 \% \mathrm{Mg}$ and $270 \mu \mathrm{m}$ in animals fed $1 \%$ cholesterol $10 \%$ fat $0.40 \% \mathrm{Mg}$ $(\mathrm{p}=0.17$ by two-way independent samples t-test, Figure $4 \mathrm{C})$. No atheromas were noted in chow-fed animals. Atherosclerosis scores for sections of the aortic arch were not significantly different between the two diet groups evaluated ( $<<0.85$ by two-sided Wilcoxon rank sum test), averaging 3.5 in animals fed $1 \%$ cholesterol $10 \%$ fat 0.11 and 3.3 in animals fed $1 \%$ cholesterol $10 \%$ fat $0.40 \% \mathrm{Mg}$ (Figure $4 \mathrm{D}$ ).
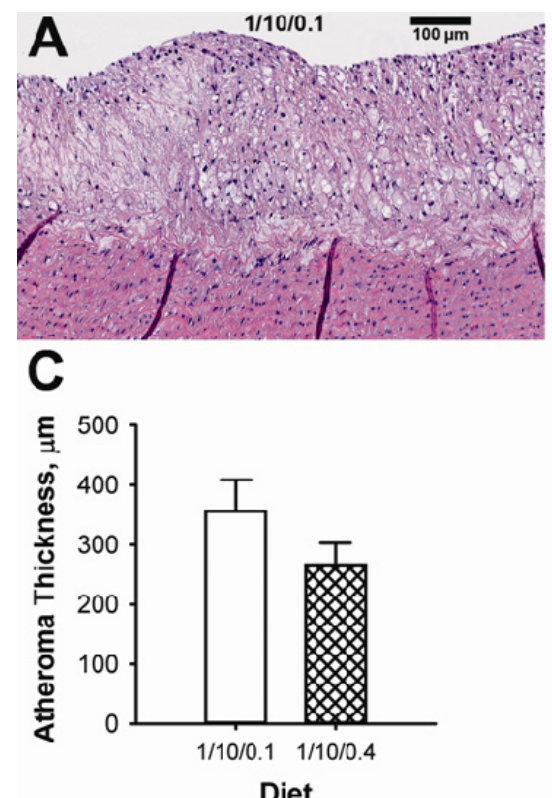

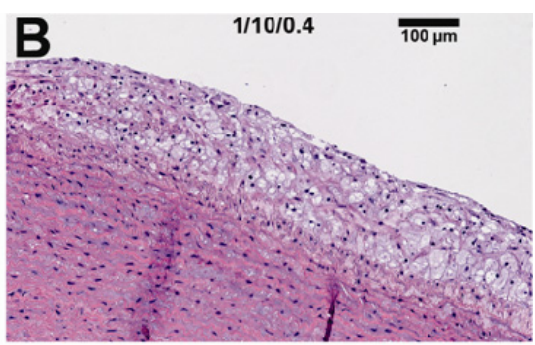

D

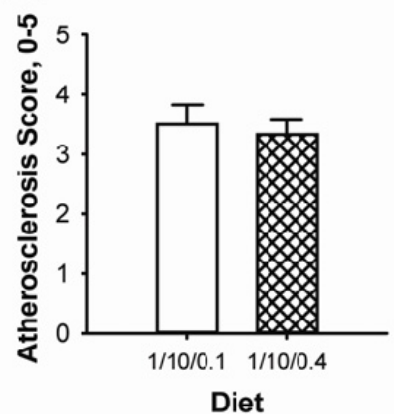

Figure 4. Development of atherosclerosis in cholesterol-fed rabbits

Rabbits were euthanized after 8 weeks on their diets and atheroma thickness (C) was measured on H\&E stained sections of formalin-fixed aortic arch tissue (A and B) using an ocular micrometer $(1 / 10 / 0.1 \mathrm{n}=8,1 / 10 / 0.4 \mathrm{n}=9$ per group). Representative images are shown at 20x magnification. Scale bars are $100 \mu \mathrm{m}$. The pathologist then assigned an atherosclerosis score (D) between 0 and 5 for each aortic arch section, with 0 indicating no evidence of atherosclerosis and 5 indicating the most severe lesion. No atheromas were noted in chow-fed controls. Bars are SEM. See Figure 1 caption for diet abbreviations. Atherosclerosis was not assessed for animals in the $2 / 20 / 0.4$ and $2 / 20 / 0.1$ groups. 


\subsection{Magnesium}

Serum $\mathrm{Mg}$ did not differ between the chow-fed animals and $1 \%$ cholesterol $10 \%$ fat $0.11 \% \mathrm{Mg}$ animals at any timepoint (Figure 5A). Serum $\mathrm{Mg}$ was significantly elevated in animals fed $1 \%$ cholesterol $10 \%$ fat $0.40 \% \mathrm{Mg}$ between $1-5$ weeks on the diet, reaching $2.6 \mathrm{mg} / \mathrm{dl}$ after one week, and then decreased to chow-fed levels in the final weeks of the study, averaging $2.1 \mathrm{mg} / \mathrm{dl}$ after eight weeks. RBC Mg decreased in all animals, and was significantly lower than chow-fed animals during weeks $2-7$ of the study for the $1 \%$ cholesterol $10 \%$ fat $0.1 \%$ $\mathrm{Mg}$ animals, and during weeks $5-7$ of the study for the $1 \%$ cholesterol $10 \%$ fat $0.40 \% \mathrm{Mg}$ group ( $<0.05$ by mixed models repeated measures ANOCOVA). After 8 weeks, chow fed animals averaged $0.025 \mathrm{mg} / \mathrm{ml}$, animals fed $1 \%$ cholesterol $10 \%$ fat $0.1 \% \mathrm{Mg}$ averaged $0.021 \mathrm{mg} / \mathrm{ml}$, and animals fed $1 \%$ cholesterol $10 \%$ fat $0.40 \% \mathrm{Mg}$ averaged $0.020 \mathrm{mg} / \mathrm{ml}$ (Figure 5B). Cardiac Mg levels after 8 weeks were highest in chow-fed animals at 0.31 $\mathrm{mg} / \mathrm{g}$ and significantly lower in both cholesterol-fed groups, with animals fed $1 \%$ cholesterol $10 \%$ fat $0.1 \% \mathrm{Mg}$ averaging $0.17 \mathrm{mg} / \mathrm{g}$ and animals fed $1 \%$ cholesterol $10 \%$ fat $0.40 \% \mathrm{Mg}$ averaging $0.15 \mathrm{mg} / \mathrm{g}$ ( $\mathrm{p}<0.05$ by Kruskal-Wallis test with Dunn's test for multiple comparisons, Figure 5C). Levels of $\mathrm{Mg}$ in skeletal muscle showed a similar pattern, with $0.31 \mathrm{mg} / \mathrm{g}$ in chow-fed animals, $0.21 \mathrm{mg} / \mathrm{g}$ in animals fed $1 \%$ cholesterol $10 \%$ fat $0.11 \% \mathrm{Mg}$ and $0.22 \mathrm{mg} / \mathrm{g}$ in animals fed $1 \%$ cholesterol $10 \%$ fat $0.40 \% \mathrm{Mg}$ ( $\mathrm{p}<0.05$ for both cholesterol-fed groups vs. chow by one-way ANOVA with Tukey's test, Figure 5D).
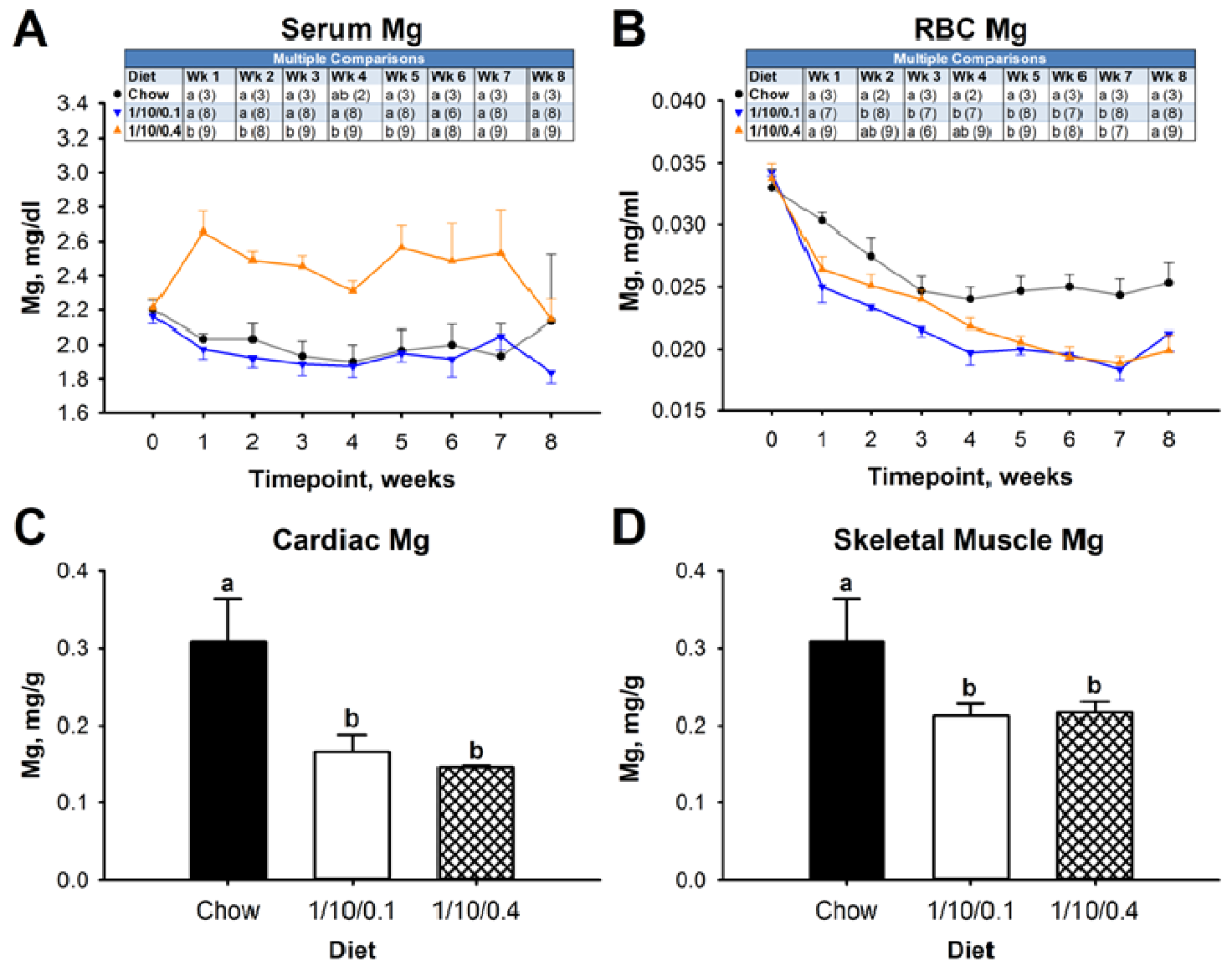

Figure 5. Magnesium $(\mathrm{Mg})$ levels in rabbits

Serum Mg levels (A) were determined on a clinical diagnostic machine, and Mg levels in red blood cells (B), cardiac (C) and skeletal muscle (D) were determined by inductively coupled plasma-optical emission spectroscopy (A and B, sample sizes per group per timepoint provided in parentheses, $\mathrm{C}$ and $\mathrm{D}$, chow $\mathrm{n}=3$, $1 / 10 / 0.1 \mathrm{n}=8$, and $1 / 10 / 0.4 \mathrm{n}=9$ ). Timepoint 0 indicates a baseline measurement prior to initiation of the atherogenic diet. Bars are SEM. See Figure 1 caption for diet abbreviations. Mg was not measured for animals in the $2 / 20 / 0.4$ and $2 / 20 / 0.1$ groups. Means not sharing a letter are significantly different $(p<0.05$ by mixed models repeated measures ANOCOVA adjusted for baseline measurements for serum and RBC Mg, by Kruskal-Wallis test for cardiac Mg and by one-way ANOVA with Tukey's test for skeletal muscle Mg). 


\section{Discussion}

In biomedical research, animal models of disease are frequently used as a platform to evaluate novel technologies or therapeutic applications. It is advantageous to provide a relevant disease model that requires a short disease development period. Here we described an improved atherogenic diet design for rabbits utilizing low dietary Mg. The diet was optimized to elevate serum cholesterol and initiate the atherosclerotic process while minimizing side effects such as jaundice and anorexia. Our results indicate that the atherogenic diet rapidly elevated blood cholesterol levels, which is a key stimulus of atherosclerosis. Evidence of atherosclerosis was also demonstrated by evaluation of $\mathrm{H} \& \mathrm{E}$ stained aortic arch sections. Animals in the $0.11 \% \mathrm{Mg}$ group displayed greater aortic arch atheroma thickness than $0.40 \% \mathrm{Mg}$-fed animals, and while this was not statistically significant, we suspect that continuation of dietary consumption for 1-2 additional weeks would lead to a greater difference between these groups. A small number of animals (2) in the $0.11 \% \mathrm{Mg}$ group died prematurely, and thus we would recommend including an additional $5-10 \%$ of animals above the required sample size to account for any loss of animals. This is typical of most animal models of disease.

Although the $2 \%$ cholesterol $20 \%$ fat diets elevated plasma cholesterol most rapidly, these rabbits also experienced a high incidence of adverse health effects including lack of appetite and jaundice. No jaundice was observed in animals fed $2 \%$ cholesterol $20 \%$ fat $0.11 \% \mathrm{Mg}$, but this may have been due to lack of feed intake or early euthanasia. Thus, we do not recommend $2 \%$ cholesterol $20 \%$ fat diets for rabbits for ethical and physiological reasons. The health complications are an important aspect of the results in this study, as this information is generally absent from similar publications. This work contributes to a more comprehensive understanding of the physiology of the cholesterol-fed rabbit that will prove important to other groups interested in utilizing this model.

The observation that low Mg status accelerates atherosclerosis suggests that Mg may be involved in regulating the health of the cardiovascular system. $\mathrm{Mg}$ is an abundant intracellular cation and is critical for bioenergetic reactions including ATP hydrolysis and protein phosphorylation. Dietary Mg may operate to reduce risk of CVD by decreasing inflammation, improving endothelial function and vascular smooth muscle tone, and preventing hypertension (Song \& Liu, 2012). An inverse correlation between Mg intake in food and water and risk of CVD has been consistently observed (Nielsen, 2010).

Serum $\mathrm{Mg}$ was elevated in rabbits consuming $0.40 \% \mathrm{Mg}$, which is expected given their higher dietary $\mathrm{Mg}$ intake. Interestingly, cardiac and skeletal muscle Mg levels in cholesterol-fed rabbits were lower than in chow-fed controls, even for rabbits fed $0.40 \% \mathrm{Mg}$, which is over twice the $0.19 \%$ present in chow. It has been previously suggested that diet-induced hypercholesterolemia leads to loss of $\mathrm{Mg}$ from tissues, generating a functional $\mathrm{Mg}$ deficiency and accelerating atherosclerosis (Altura et al., 1990). Our results support this hypothesis, and also highlight the important point that serum $\mathrm{Mg}$ levels may not reflect true cellular, physiological and functional $\mathrm{Mg}$ status (Reinhart, 1988).

We also chose to measure vWF, a biomarker of endothelial damage, using our in-house validated assay (Smith et al., 2010). vWF is produced in and secreted from vascular endothelial cells and is involved in platelet plug formation for wound healing (Sadler, 1998). Elevated circulating vWF predicts development of coronary artery disease in healthy subjects, and also predicts risk of myocardial infarction in patients with existing atherosclerosis (Spiel et al., 2008). We expected vWF to be elevated in cholesterol-fed rabbits. vWF levels were variable in this study but were generally higher in rabbits consuming cholesterol.

The rabbit is one of several animal models used to study atherosclerosis. Others include genetically modified mice (Getz \& Reardon, 2006), rats (Herrera \& Ruiz-Opazo, 2005), monkeys (Ross \& Harker, 1976), pigs (Koskinas et al., 2010), guinea pigs (Fernandez \& Volek, 2006), hamsters (Russell \& Proctor, 2006) and gerbils (Nicolosi et al., 1981). Rabbits have certain advantages that allow them to remain a useful model. Their size, intermediate between pigs and rodents, provides the researcher with larger organs and blood vessels while remaining practical to handle. Like humans, cholesterol-fed rabbits carry most blood cholesterol in LDL (Smith et al., 2012), as opposed to many genetically modified mouse models in which very low-density lipoprotein (VLDL) is the primary blood lipoprotein fraction.

\section{Conclusion}

In conclusion, we evaluated atherogenic diets with varying amounts of cholesterol, fat and $\mathrm{Mg}$. The $1 \%$ cholesterol $10 \%$ fat $0.11 \% \mathrm{Mg}$ diet was optimal, generating plasma cholesterol levels above $1000 \mathrm{mg} / \mathrm{dl}$ in only 5 weeks and the greatest aortic arch atheroma thickness while minimizing health complications for the animals. Reducing dietary $\mathrm{Mg}$ below chow levels, even by only $0.08 \% \mathrm{w} / \mathrm{w}$, was a means of accelerating atherosclerosis. This acceleration of atherosclerosis maximizes cost-efficiency and rapidly delivers results. The phenotypic data 
presented here will be useful for researchers using the cholesterol-fed rabbit model to study CVD.

\section{Acknowledgements}

This work was supported by NIH R37 EB002641. The authors acknowledge D.G. Simpson for helpful statistical discussion, R. Abuhabsah for assistance with experimental procedures, and Provena Covenant Medical Center and the University of Illinois Veterinary Diagnostic Laboratory for assistance with histology.

\section{References}

Altura, B. T., Brust, M., Bloom, S., Barbour, R. L., Stempak, J. G., \& Altura, B. M. (1990). Magnesium dietary intake modulates blood lipid levels and atherogenesis. Proceedings of the National Academy of Sciences of the United States of America, 87, 1840-1844. Retrieved from http://www.ncbi.nlm.nih.gov/pubmed/2308944

Fernandez, M. L., \& Volek, J. S. (2006). Guinea pigs: a suitable animal model to study lipoprotein metabolism, atherosclerosis and inflammation. Nutrition and Metabolism, 3, 17. Retrieved from http://www.ncbi.nlm.nih.gov/pubmed/16566831

Finking, G., \& Hanke, H. (1997). Nikolaj Nikolajewitsch Anitschkow (1885-1964) established the cholesterol-fed rabbit as a model for atherosclerosis research. Atherosclerosis, 135, 1-7. Retrieved from http://www.ncbi.nlm.nih.gov/pubmed/9395267

Getz, G. S., \& Reardon, C. A. (2006). Diet and murine atherosclerosis. Arteriosclerosis, Thrombosis, and Vascular Biology, 26, 242-249. Retrieved from http://www.ncbi.nlm.nih.gov/pubmed/16373607

Hansson, G. K., \& Hermansson, A. (2011). The immune system in atherosclerosis. Nature Immunology, 12, 204-212. Retrieved from http://www.ncbi.nlm.nih.gov/pubmed/21321594

Herrera, V. L., \& Ruiz-Opazo, N. (2005). Genetic studies in rat models: insights into cardiovascular disease. Current Opinion in Lipidology, 16, 179-191. Retrieved from http://www.ncbi.nlm.nih.gov/pubmed/15767858

King, J. L., Miller, R. J., Blue, J. P. Jr., O'Brien, W. D. Jr., \& Erdman, J. W. Jr. (2009). Inadequate dietary magnesium intake increases atherosclerotic plaque development in rabbits. Nutrition Research, 29, 343-349. Retrieved from http://www.ncbi.nlm.nih.gov/pubmed/19555816

Koskinas, K. C., Feldman, C. L., Chatzizisis, Y. S., Coskun, A. U., Jonas, M., \& Maynard, C. (2010). Natural history of experimental coronary atherosclerosis and vascular remodeling in relation to endothelial shear stress: a serial, in vivo intravascular ultrasound study. Circulation, 121, 2092-2101. Retrieved from http://www.ncbi.nlm.nih.gov/pubmed/20439786

Kumar, V., Fausto, N., \& Abbas, A. K. (2004). Robbins and Cotran Pathologic Basis of Disease (7th ed.). Saunders, Philadelphia, PA.

Nicolosi, R. J., Marlett, J. A., Morello, A. M., Flanagan, S. A., \& Hegsted, D. M. (1981). Influence of dietary unsaturated and saturated fat on the plasma lipoproteins of Mongolian gerbils. Atherosclerosis, 38, 359-371. Retrieved from http://www.ncbi.nlm.nih.gov/pubmed/7225175

Nielsen, F. H. (2010). Magnesium, inflammation, and obesity in chronic disease. Nutrition Reviews, 68, 333-340. Retrieved from http://www.ncbi.nlm.nih.gov/pubmed/20536778

Ouchi, Y., Tabata, R. E., Stergiopoulos, K., Sato, F., Hattori, A., \& Orimo, H. (1990). Effect of dietary magnesium on development of atherosclerosis in cholesterol-fed rabbits. Arteriosclerosis, Thrombosis, and Vascular Biology, 10, 732-737. Retrieved from http://www.ncbi.nlm.nih.gov/pubmed/2403301

Reinhart, R. A. (1988). Magnesium metabolism. A review with special reference to the relationship between intracellular content and serum levels. Archives of Internal Medicine, 148, 2415-2420. Retrieved from http://www.ncbi.nlm.nih.gov/pubmed/3056314

Roger, V. L., Go, A. S., Lloyd-Jones, D. M., Adams, R. J., Berry, J. D., \& Brown, T. M. (2011). Heart disease and stroke statistics--2011 update: a report from the American Heart Association. Circulation, 123, e18-e209. Retrieved from http://www.ncbi.nlm.nih.gov/pubmed/21160056

Ross, R., \& Harker, L. (1976). Hyperlipidemia and atherosclerosis. Science, 193, 1094-1100. Retrieved from http://www.ncbi.nlm.nih.gov/pubmed/822515

Russell, J. C., \& Proctor, S. D. (2006). Small animal models of cardiovascular disease: tools for the study of the roles of metabolic syndrome, dyslipidemia, and atherosclerosis. Cardiovascular pathology : the official 
journal of the Society for Cardiovascular Pathology, 15, 318-330. Retrieved from http://www.ncbi.nlm.nih.gov/pubmed/17113010

Sadler, J. E. (1998). Biochemistry and genetics of von Willebrand factor. Annual Review of Biochemistry, 67, 395-424. Retrieved from http://www.ncbi.nlm.nih.gov/pubmed/9759493

Smith, B. W., Simpson, D. G., Sarwate, S., Miller, R. J., Blue, J. P. Jr., \& Haak, A. (2012). Contrast ultrasound imaging of the aorta alters vascular morphology and circulating von Willebrand Factor in hypercholesterolemic rabbits. Journal of Ultrasound in Medicine, 31, 711-720. Retrieved from http://www.ncbi.nlm.nih.gov/pubmed/22535718

Smith, B. W., Strakova, J., King, J. L., Erdman, J. W. Jr., \& O'Brien, W. D. Jr. (2010). Validated sandwich ELISA for the quantification of von Willebrand factor in rabbit plasma. Biomarker Insights, 5, 119-127. Retrieved from http://www.la-press.com/validated-sandwich-elisa-for-the-quantification-of-von-willebrand-fact-article-a23 70 -abstract

Song, Y., \& Liu, S. (2012). Magnesium for cardiovascular health: time for intervention. The American Journal of Clinical Nutrition, 269-270. Retrieved from http://www.ncbi.nlm.nih.gov/pubmed/22218155

Spiel, A. O., Gilbert, J. C., \& Jilma, B. (2008). von Willebrand factor in cardiovascular disease: focus on acute coronary syndromes. Circulation, 117, 1449-1459. Retrieved from http://www.ncbi.nlm.nih.gov/pubmed/18347221 